\title{
A Novel Illumination Distribution Arrangement for Indoor VLC Using 17 Locations of Light Source
}

\author{
Mohammed Musa Mohammed Musa, Hongbing Qiu \\ Department of Communication, Institute of Information and Communication, Guilin University of Electronics Technology, Guilin, China
}

Email address:

musait a,hotmail.com(M. M. M. Musa),609016768@qq.com(Hongbing Qiu)

\section{To cite this article:}

Mohammed Musa Mohammed Musa, Hongbing Qiu. A Novel Illumination Distribution Arrangement for Indoor VLC Using 17 Locations of Light Source. Advances in Wireless Communications and Networks. Vol. 4, No. 1, 2018, pp. 5-10. doi: 10.11648/j.awcn.20180401.12

Received: June 9, 2018; Accepted: August 17, 2018; Published: September 10, 2018

\begin{abstract}
In the recent years Visible Light Communication (VLC) has gained great interest due to the rapid developments in Light Emitting Diodes (LEDs) fabrication. Efficiency, durability and long life span of LEDs make them a promising residential lighting equipment as well as an alternative cheap and fast data transfer equipment. The advantages of indoor VLC that is offering illumination and data transmission simultaneously using the existing infrastructure, White LED lamp used as source. The significant challenge is a right location of the source allowed users to receive fine illumination as well as high data rate. In this paper we proposed a numerous distribution patterns with different number of LEDs to increase the signal to noise ratio (SNR) and enhanced the uniformity of the illumination. The indoor VLC system is modeled using the proposed pattern and the theoretical analysis is presented. Firstly we compare the normal distribution of the luminous intensity of one locations LED located on the center of room ceiling with our four locations LEDs model. To eliminate spot lighting 8 lactation of LED lamps was proposed. Lastly our novel arrangement using 17 locations has been investigated. A proposed design provide normal distribution which enhance the lighting and communication in whole room. A mathematical model of illumination LEDs has been studied, as well as the basic parameters which are important for the development of the design.
\end{abstract}

Keywords: Indoor VLC, LED Lamp, Lighting, Illumination

\section{Introduction}

Wireless communication is dominated by RF (Radio Frequency) communication, since James Maxwell and Michael Faraday demonstrated that Electromagnetic wave travels in a straight line through space. Radio frequency spectrum for wireless communication in between $3 \mathrm{kHz}$ to $300 \mathrm{GHz}$, and every portion of this frequency spectrum is already in use one way or the other which has resulted in severe congestion of existing radio frequency spectrum [1]. One key of the challenges of any wireless operator is to improve indoor capacity, because the most cellular traffic is produce indoor, which are used density, which congest the traffic and the flow of data become slow. To meet the required data rate we have to use the spectrum in most efficient method, [2] moreover RF frequency has other limitation, such as, high frequency effect human body, electromagnetic interference, the implementation of the RF equipment and maintained them are expansive, license fees.. ect. [3].
An alternative method of communication of wireless communication using visual light is visible light communication, it's used emitting light source and light sensor receiver for data transfer. The dominant device for transmitter is Light-emitting diodes (LEDs) and for receiver is photo diode. This method have several advantages such as safety of human health, faster switching time and greater design flexibility [4], used is without license, any existing light source such as room illuminations and displays could be used as transmitters, in other word it can be used at a place where radio waves cannot be used, for example hospitals or area around precision machine [5]. moreover the light signal cannot penetrate the wall which provide more security[6]. For long-distance communications Line-of-Sight (LoS) is essential that the sender and the receiver must see each other directly. Stay of signals in the room or office prevents possibility of any interference in adjacent rooms or offices, by this way each room will constitute a cell and the capacity productivity will rise to the top levels. The equipment used is cheaper when compared with RF devices. [7] 
LED have been widely deployed for illumination due to their high performance and energy efficiency properties compared with conventional incandescent and fluorescent lamps. In 2011 light-fidelity (Li-Fi) was introduced by Haas [7]. Although there has been significant progress in this area in the past decade but still there are some challenges, to locate the source on the ceiling in order to emit light to cover all the floor of the room is the main of this challenges [8].

\section{System Description}

\subsection{System Link}

Indoor VLC system is like other communication system in transmitting information from a transmitter (light emitting diode) to a receiver (photo diode) through the construction of a time-varying physical quantity or a signal channel (line of sight). In each communication system, the transmitted signal is corrupted by degradation due the environment surrounded, our proposed system is design to give minimum noise and interference, which the signaling processing and modulation to give data reliably and at high rates over these distorting channels are used.

\subsection{VLC Communication System}

Recently VLC communication system is take place as promise technology for wireless optical communication. The signals in the $380-780 \mathrm{~nm}$ wavelength interval of the electromagnetic spectrum are the light signals that can be detected by the human eye. It is possible to achieve illumination and data transfer simultaneously by means of LEDs that is the prominent lighting equipment lately. By this way both interior lighting of a room and data transfer will be achieved without the need of an additional communication systems. As in [8] basic configuration of a VLC communication system is given in Figure 1.

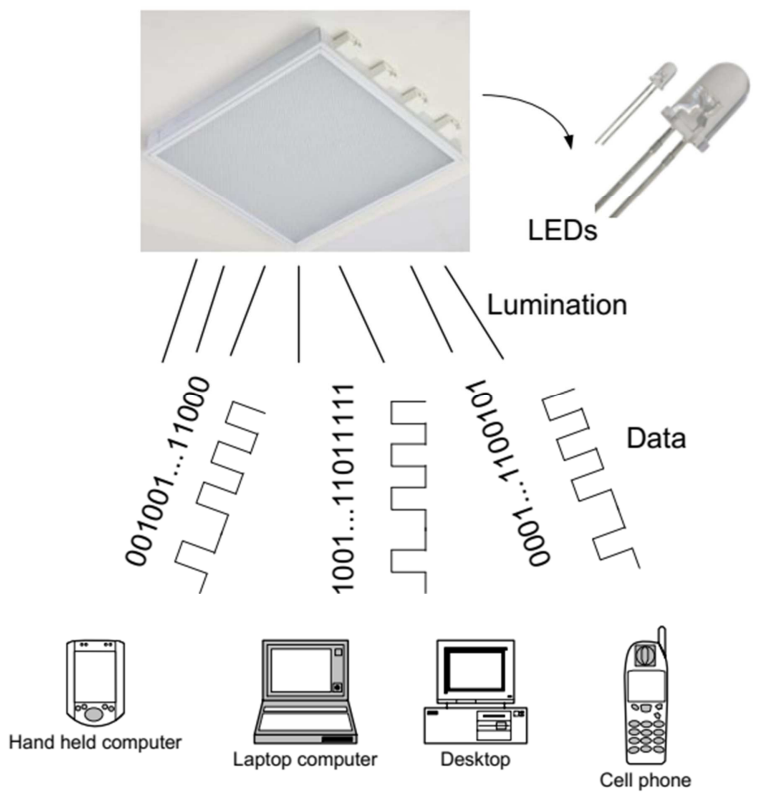

Figure 1. Basic VLC configuration.
In a VLC system, the receiver may be located far from the LED lamp where the SNR is much smaller than at those locations close to the lamp. The lower SNR is obtained as the distance from the source increases and as the incident angle increases [9] to improve the performance of the VLC system across the entire room. The SNRs the receiver plane are analyzed. For simplicity, the reflections of walls are not considered in analyzing the SNR since the light from the line-of-sight (LOS) is dominant [10].

\section{System Model}

\subsection{LED Light Properties}

In light there are two properties, a luminous intensity and a transmitted optical power. The relationship between photometric and radiometric quantities is explained in [8], in this paper we concentrate on the luminance in order to give normal distribution.

\subsection{Illuminance of Led Lighting}

The illuminance expresses the brightness of an illuminated surface, in this paper we study the distribution of illuminance at a desk surface. The luminous intensity in angle:

$$
I(\Phi)=I(0) \cos ^{m}(\Phi)
$$

A horizontal illumination $\mathrm{E}$ is given by

$$
E=\frac{I(0) \cos ^{m}(\Phi)}{d^{2}} \cdot \cos (\psi)
$$

Where $m$ is Lambertian order given by the angle of semi power as $m=-\ln 2 / \ln \left(\cos \Phi_{1 / 2}\right), \mathrm{I}(0)$ is the center luminous intensity of an LED, $\Phi$ is the angle of irradiance with respect to the transmitter perpendicular axis, $\psi$ is the angle of incidence related to the receiver perpendicular axis.

The illuminance of light is standardized by International Organization for Standardization (ISO) in the range between 300 to $1500 \mathrm{~lx}$ for indoor office work.

\subsection{Received Power of Directed Light}

In this paper we assume the receiver are stationery, in this case LOS will be simultaneously present at the receiver. Thus, directed LOS-channel is given as:

$$
H_{L O S}(0)=\left\{\begin{array}{cc}
\frac{(1+m) A}{2 \pi d^{2}} \cos ^{m}(\Phi) T_{s}(\psi) g(\psi) \cos (\psi) & 0 \leq \psi \leq \Psi_{c} \\
0 & \psi>\Psi_{c}
\end{array}\right.
$$

Where A is the physical area of the photo diode (PD), $d$ the distance between transmitter and receiver, $\operatorname{Ts}(\psi)$ is gain of optical receiver, $\mathrm{g}(\psi)$ is the optical concentrator gain, $\Psi_{c}$ is the optical concentrator field-of-view (FOV).

The received optical power $P r$ is derived by the transmitted light power $P t$, as follows 


$$
P_{r}=H(0) \cdot P_{t}
$$

\section{System Design}

In our proposed design, we consider the basic parameters as in [10] A room was assumed for the purpose of these analyses. An indoor VLC system model with the size of 5.0 $\mathrm{m} \times 5.0 \mathrm{~m} \times 3.0 \mathrm{~m}$ is established for following research. Fixtures in the room were arranged as shown in Figure 1. The LED lights are installed on the ceiling at a height of $2.5 \mathrm{~m}$. The receiver is located at a plane at the height of $0.85 \mathrm{~m}$. Intensity modulation and direct detection are used in the system. Ceiling, floor and walls are treated as the reflected surfaces. Other parameters relevant to the system are listed in table 1 . we study different scenarios of source locations start from the 1, 4, 8 and 17 locations. The semi-angle at halfpower of an LED chip is 70 deg., the center luminous intensity of an LED chip is $0.73 \mathrm{~cd}$, respectively. The transmitted optical power of an LED chip is $20.0 \mathrm{~mW}$. Those conditions summarized in Table 1.

Table 1. System parameters.

\begin{tabular}{ll}
\hline Transmitted optical power & $\mathbf{2 0}[\mathbf{m W}]$ \\
\hline Semi-angle at half power & $70[\mathrm{deg}]$. \\
Center luminous intensity & $0.73[\mathrm{~cd}]$ \\
FOV at a receiver & $60[\mathrm{deg}]$. \\
detector physical area of a PD & $1.0[\mathrm{~cm} 2]$ \\
gain of an optical filter & 1.0 \\
refractive index of a lens at a PD & 1.5 \\
O/E conversion efficiency & $0.53[\mathrm{~A} / \mathrm{W}]$ \\
\hline
\end{tabular}

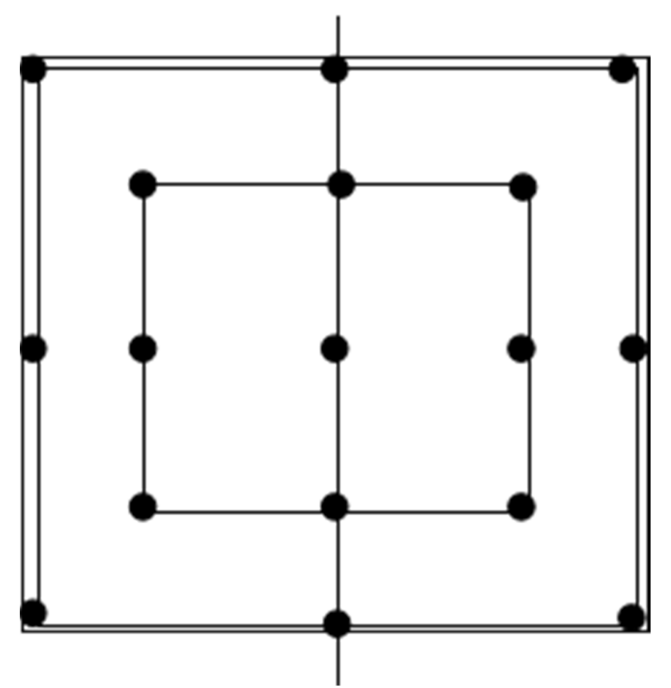

Figure 2. The model room shows the novel arrangement of 17 locations of LEDs on the roof. The room size is $5 \mathrm{~m} \times 5 \mathrm{~m} \times 3 \mathrm{~m}$.

\section{Simulation Results}

\subsection{Simulation Result for One LED Location}

In our simulation, firstly the simulation of the one LED light located in the center of the ceiling at coordinate of [2.5, $2.5,3.0]$ are investigated as [5], a simulation resulted clearly that the light is concentrate on the center of the ceiling which cause low illumination as well as data rate at the corners of the room.

3D PIot for Room SNR Distribution of LOS

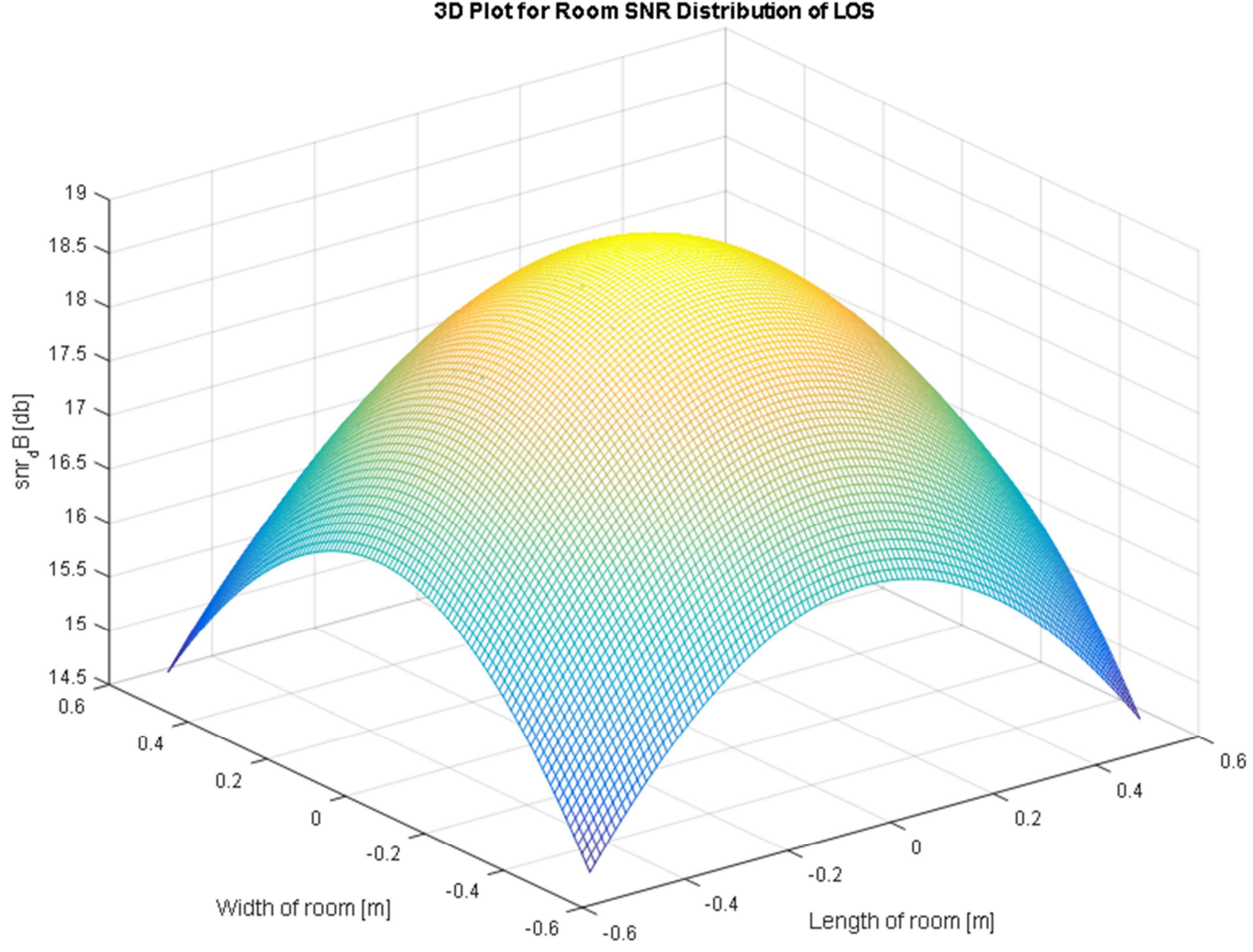

Figure 3. Illuminance distribution in case of One transmitter. 
Normal distribution of the luminous intensity

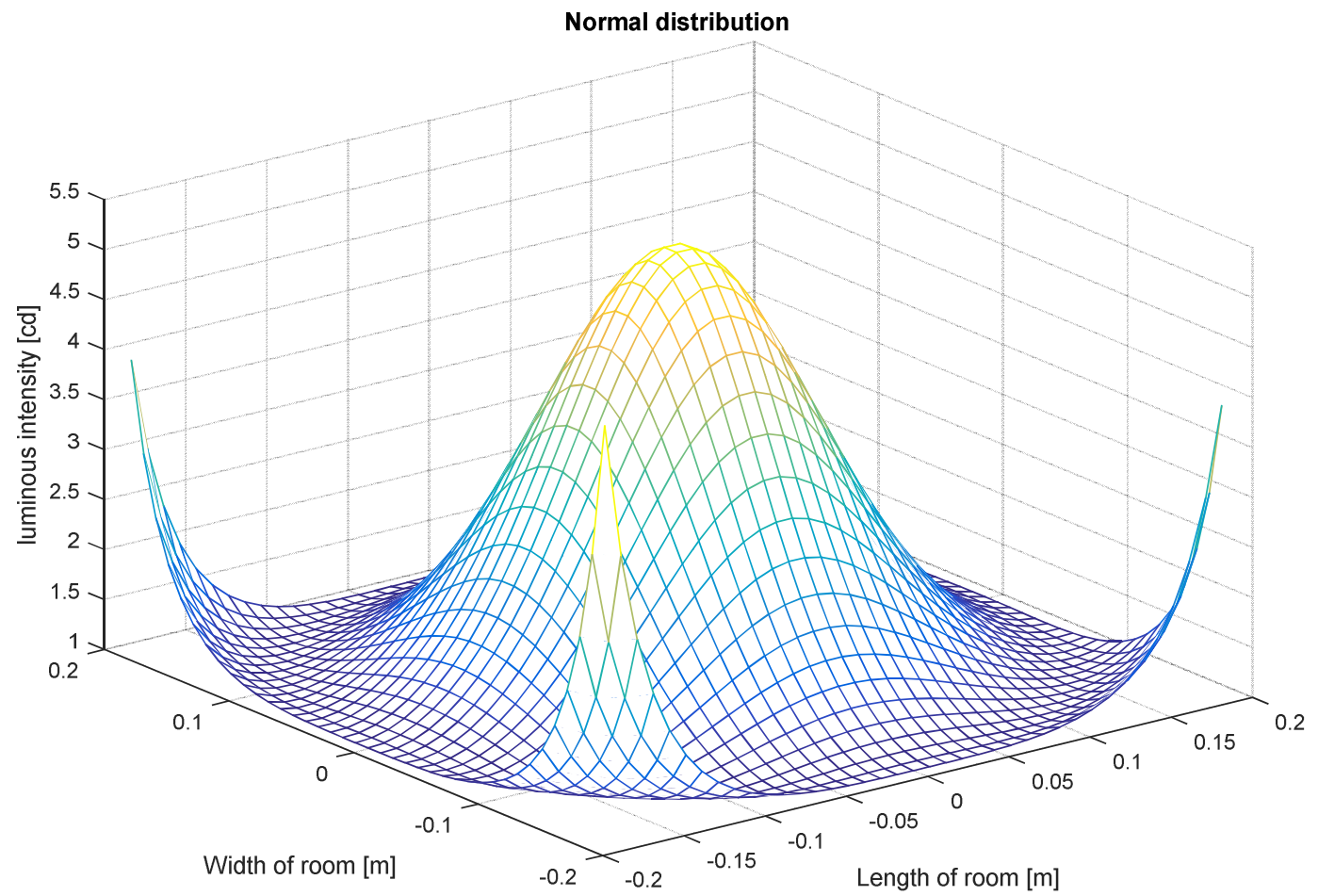

Figure 4. Normal distribution of the luminous intensity of One transmitter.

\subsection{Simulation Result for Four Led Locations}

The system has been improves by adding four sources of LEDs in different locations to enhance the illumination and power distribution located at the coordinate of $(1.25,1.25)$,
$(3.75,1.25),(3.75,1.25)$, and $(3.75,3.75)$, arrangement has been done according to room diameter of the room mentioned above, led source is located in each corner.

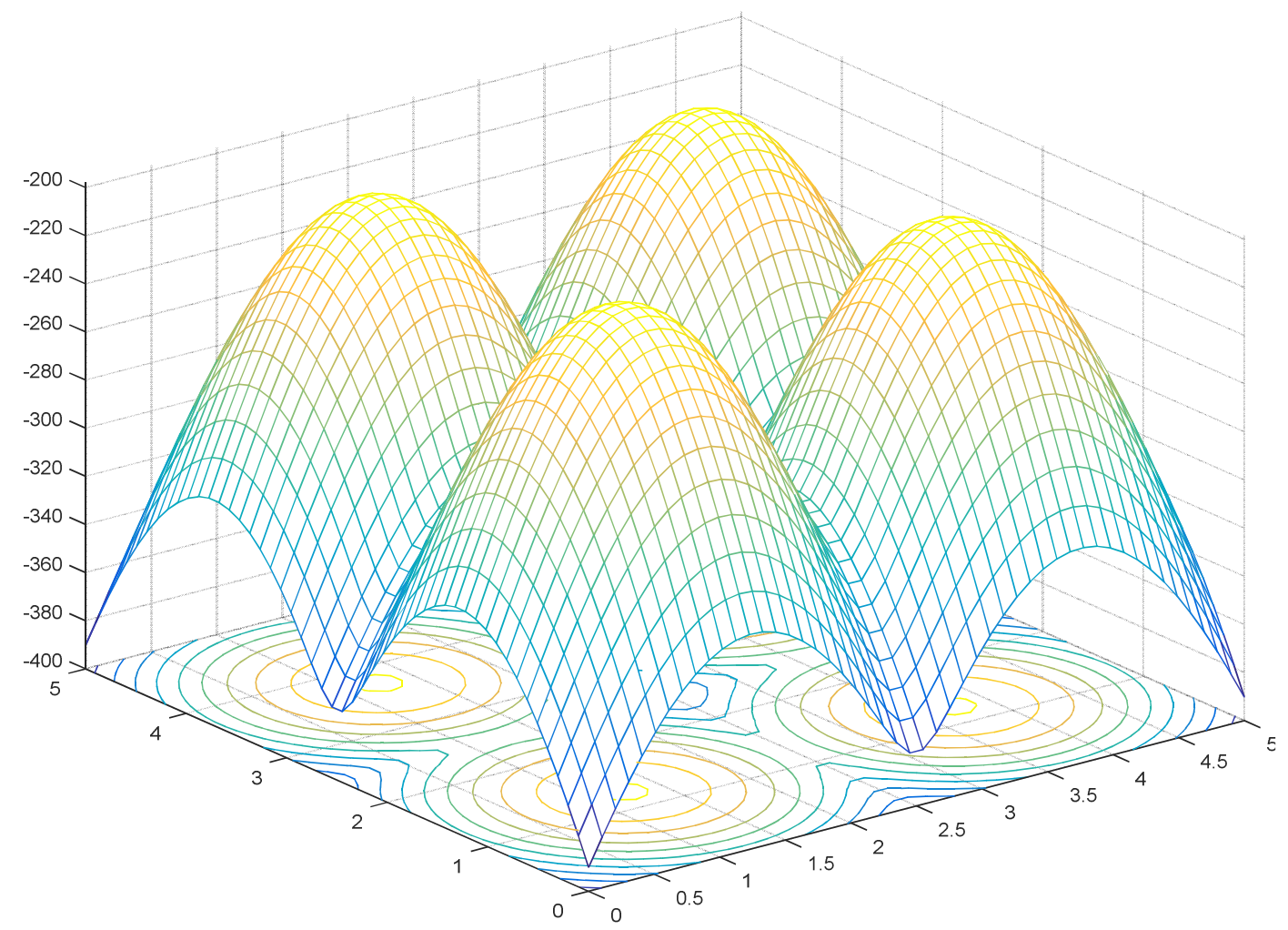

Figure 5. Power distribution in case of 4 transmitters. 
The result in case of four LEDs light arrangement it's clear that the light is focus in spot area directly under the location of LED light source other space receive weak signal and dim light too.

\subsection{Simulation result for Eight Led Locations}

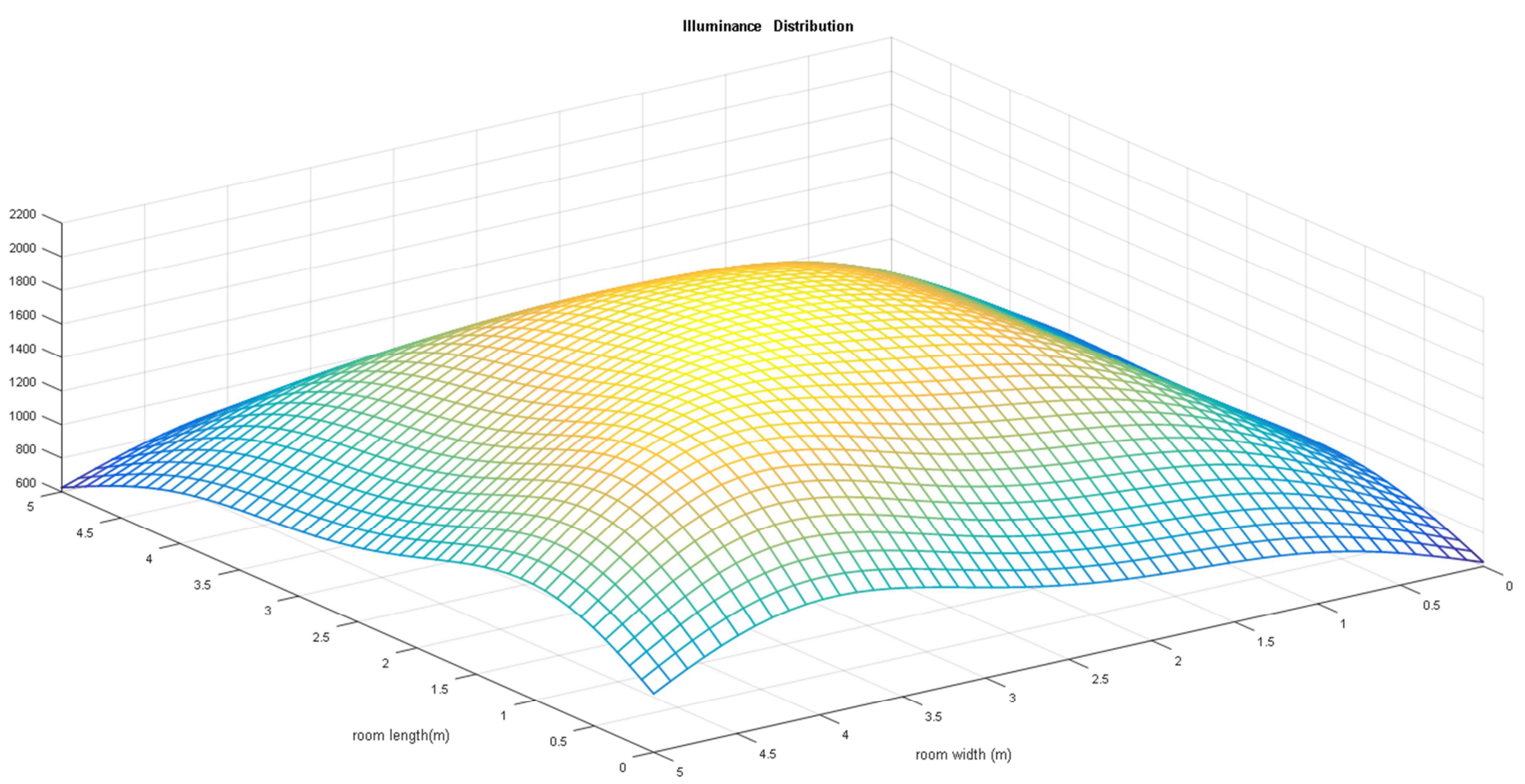

Figure 6. The distribution of illuminance. Min. 624.2 lx, Max. 1888.6 lx, Ave. 1,256.4 lx.

Figure 6 shows the distribution of horizontal illuminance using 8 locations of LEDs arranged at ceiling as $[0.8,0.8$, 2.15], [0.2, 4.8, 2.15], [4.8, 0.2, 2.15], [4.8, 4.8, 2.15], [1.7, $1.7,2.15],[1.7,3.4,2.15],[3.4,1.7,2.15],[3.4,3.4,2.15]$, a user terminal equipped with the LED lights listed in Table I. From this figure, the maximum illuminance is $1888.6 \mathrm{~lx}$, the minimum illuminance is $6242.4 \mathrm{~lx}$, and the average illuminance is $1.256 .4 \mathrm{~lx}$ which is in the range of the ISO standard, the light is obtained in all the places of the room. Therefore, this result shows that this LED lighting has function as lighting.

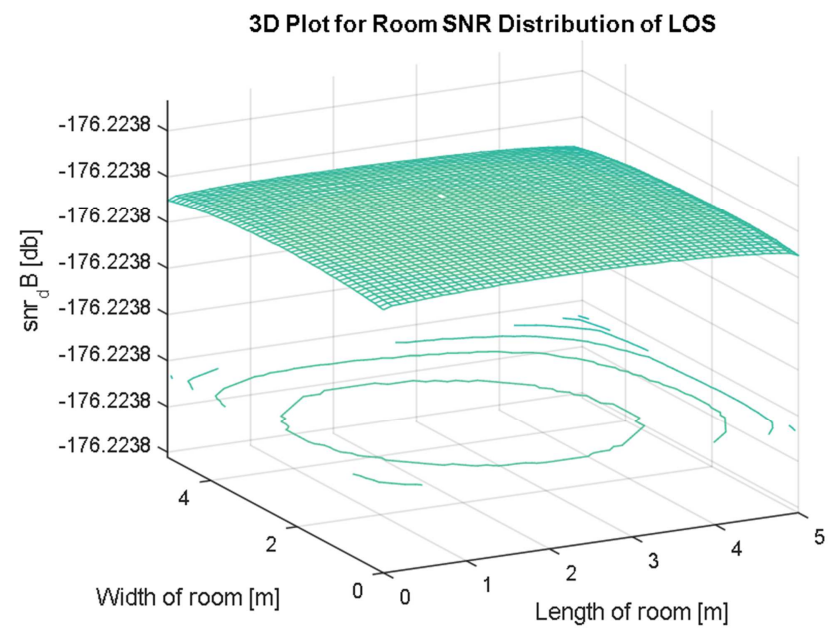

Figure 7. The normal distribution of received power.
In the table 1 we analyses the parameters of FOV is 60.0 deg., and the physical detection area of a PD is $1.0 \mathrm{~cm}^{2}$. The gain at an optical filter is 1.0 , and the refractive index of an optical concentrator is 1.5 . The $\mathrm{O} / \mathrm{E}$ conversion efficiency of a PD is $0.53 \mathrm{~A} / \mathrm{W}$, and a silicon PD whose peak sensitivity is in visible wavelength is assumed.

\subsection{Simulation Result for 17 Led Locations}

For more arrangements to optimize novel location of leds as in figure 7,17 leds has been investigated and located as $(0.2,0.2),(0.2,2.5),(0.2,4.8),(2.5,0.2),(4.8,0.2),(1.25$, $1.25),(2.5,1.25),(3.75,1.25),(1.25,2.5),(1.25,3.75),(2.5$, $2.5),(3.75,2.5),(2.5,3.75),(2.5,4.8),(4.8,2.5),(3.75,3.75)$, $(4.8,4.8)$. on the ceiling of the room to receive normal distribution power. From considerations of uniformity, lux level on the playing surface, and the wattage of the light source, the arrangement is chosen to be placed at a height of $2.15 \mathrm{~m}$ for all scenarios. Figure 7 is the Illuminance plot of the simulated model for this arrangement, the result show the novel arrangement give normal distribution better than the first scenario.

\section{Conclusion}

LED as lighting and data transmission are proposed with different scenarios in our design, firstly; we investigate the pervious work in this area used one Led located in the center of ceiling give result clearly showed low illumination as well as date rate in the corners of the room. The system design has 
been improved used four leds in different locations which resulted spot light directly under the location of the transmitter and dim with low data rate in other area more and more improvement was done to enhance the light beside the increase the data rate of the signal by using eight different location in ceiling which cover all room area and meet the ISO of lighting standard.

Our novel optimize arrangement of led's location used minimum numbers leds light lamp an give normal distribution meet the iso standard, provide high data rate which mean better communication and light in all part of the room, which declare that best uniform power distribution.

\section{Acknowledgements}

This research was supported by the project of National Natural Science Foundation of China (No.61761014) and GUET Excellent Graduate Thesis Program (16YJPYBS02).

\section{Conflicts of Interest}

The authors declare that they have no conflicts of interest.

\section{References}

[1] R. Sharma, A. C. Kumari, M. Aggarwal and S. Ahuja, "Performance analysis of LED based indoor VLC system under receiver mobility," 2017 International Conference on Computing, Communication and Automation (ICCCA), Greater Noida, 2017, pp. 945-950.

[2] Steve Hranilovic. "Wireless Optical Communication Systems" eBook ISBN: 0-387-22785-7. Springer Science + Business Media, Inc. Boston, US, 2005.
[3] Dr. Mahendra Kumar., "Advantages and Disadvantages of Radio Frequency", INDIAN JOURNAL OF APPLIED RESEARCH, ISSN - 2249-555X, Volume 5, Issue:10, October 2015.

[4] Dehao $\mathrm{Wu}$, Wen-De Zhong "Short-range visible light ranging and detecting system using illumination light emitting diodes", IET Optoelectronics, ISSN 1751-8768, November 2017.

[5] Taner Cevik and Serdar Yilmaz. "An overview of visible light communication systems", International Journal of Computer Networks \& Communications (IJCNC) Vol. 7, No. 6, November 2015.

[6] X. Song, Y. Wei, Z. Zhao and M. Wang, "The influence of driving current on the LED modulation bandwidth indoor VLC," 2017 IEEE/CIC International Conference on Communications in China (ICCC Workshops), Qingdao, 2017, pp. 1-6.

[7] Moussa Ayyash, Hany Elgala, Abdallah Khreishah "Coexistence of $\mathrm{WiFi}$ and $\mathrm{LiFi}$ Toward 5G: Concepts, Opportunities, and Challenges", IEEE IEEE Communications Magazine, vol 54, Issue:2, pp. 64 - 71, February 2016.

[8] Steven De Lausnay, Lieven De Strycker, Jean-Pierre, Bart Nauwelaers "Matlab based Platform for the Evaluation of Modulation Techniques used in VLC", 12th International Conference on DEVELOPMENT AND APPLICATION SYSTEMS, Suceava, Romania, May 15-17, 2014.

[9] Anil Yesiljaya, Farshad Miramirkhani, Ertugrul Basar, Erdal Panayirci, Murat Uysal." Performance of MIMO Enhanced Unipolar OFDM with Realistic Indoor VL channel models", IEEE, journal No. 113E307, 2016.

[10] W. Tang et al., "Analysis of indoor VLC positioning system with multiple reflections," 2017 16th International Conference on Optical Communications and Networks (ICOCN), Wuzhen, 2017, pp. 1-3. 